\title{
Solid Papillary Carcinoma and Encapsulated Papillary Carcinoma of the Breast: Clinical-Pathologic Features and Basement Membrane Studies of 50 Cases
}

\author{
Sarah Morgan ${ }^{a}$ David Dodington ${ }^{a}$ Jessie M. Wu ${ }^{a, b}$ Gulisa Turashvilia, b \\ aDepartment of Laboratory Medicine and Pathobiology, University of Toronto, Toronto, ON, Canada; ${ }^{b}$ Department \\ of Pathology and Laboratory Medicine, Mount Sinai Hospital, Toronto, ON, Canada
}

\section{Keywords}

Solid papillary carcinoma - Encapsulated papillary carcinoma · Basement membrane · Collagen IV · Laminin · Immunohistochemistry

\begin{abstract}
Introduction: Solid papillary carcinoma (SPC) and encapsulated papillary carcinoma (EPC) of the breast are usually considered in situ lesions due to favorable prognosis, despite the variable presence of myoepithelial cells. We aimed to describe clinical-pathologic features including basement membrane (BM) studies in these tumors. Methods: Patients diagnosed with SPC and EPC in 2000-2019 were retrospectively identified. Microscopic slides and clinical history were reviewed. Immunohistochemical stains for BM and myoepithelial markers were performed. Results: Of 23 SPCs and 27 EPCs, there were 5/23 (21.7\%) pure SPCs and 9/27 (33.3\%) pure EPCs, while 4/23 (17.4\%) and 12/27 (44.5\%) were associated with ductal carcinoma in situ (DCIS), and 6/23 (26.1\%) and $6 / 27$ (22.2\%) with invasive carcinoma, respectively; $8 / 23$ (34.8\%) SPCs were considered invasive. The median tumor size was $1.7 \mathrm{~cm}$ (range 0.1-16). All tumors were positive for hormone receptors and negative for HER2. Myoepithelial
\end{abstract}

cells were absent in 20 tumors (40\%) and focally present in 30 (60\%). Collagen IV and laminin were negative in most invasive lesions, but they were expressed in 21/21 (100\%) and $18 / 21(85.7 \%)$ of EPCs without invasion, and 16/17 (94.1\%) and 10/17 (58.8\%) SPCs, including invasive SPCs, respectively. Lymph node involvement was identified in 3/26 (11.5\%) patients, including micrometastasis in $1 \mathrm{EPC}$ associated with DCIS, macrometastasis in 1 EPC associated with invasive carcinoma, and isolated tumor cells in 1 invasive SPC. Of 31 patients with outcome data (median follow-up 35 months, range 1-85), 2 (6.5\%; 1 SPC, 1 EPC) developed local recurrence, both associated with invasive carcinoma. No distant recurrences or deaths were observed. Conclusions: Our study confirms favorable prognosis of SPCs and EPCs, with 2 local recurrences occurring in the presence of invasion. SPCs are more commonly associated with invasive carcinoma or considered invasive compared to EPCs (60.9 vs. $22.2 \%)$. The presence of BM material and lack of lymph node involvement in most cases indicates that the majority of these tumors may represent in situ lesions; however, some may behave as low-grade invasive malignancy with metastatic potential even in the absence of conventional invasion.

(c) 2021 The Author(s)

Published by S. Karger AG, Basel karger@karger.com www.karger.com/pat

Karger $\stackrel{\text { ' }}{5}$

GOPEN ACCESS
(C) 2021 The Author(s)

Published by S. Karger AG, Basel

This is an Open Access article licensed under the Creative Commons Attribution-NonCommercial-4.0 International License (CC BY-NC) (http://www.karger.com/Services/OpenAccessLicense), applicable to the online version of the article only. Usage and distribution for commercial purposes requires written permission.
Correspondence to:

Gulisa Turashvili, gulisa.turashvili@ sinaihealth.ca 


\section{Introduction}

Papillary lesions of the breast encompass a heterogeneous group ranging from benign to malignant entities. These lesions are defined morphologically based on the presence of fibrovascular cores lined by epithelial cells, and include intraductal papillomas with or without atypical epithelial proliferation, papillary ductal carcinoma in situ (DCIS), and papillary carcinomas [1]. Atypical epithelial proliferations may focally involve intraductal papillomas and include atypical ductal hyperplasia $(<3 \mathrm{~mm})$ or DCIS $(\geq 3 \mathrm{~mm})$. Papillary DCIS exhibits myoepithelial cells at the periphery but not around the fibrovascular cores. Papillary carcinomas account for $0.5-1 \%$ of breast cancers and include solid papillary carcinoma (SPC), encapsulated papillary carcinoma (EPC), and invasive papillary carcinoma [2-4]. SPC is composed of well-defined nodules with delicate fibrovascular cores, while EPC usually presents as one or more well-circumscribed nodules within a cystically dilated duct exhibiting well-developed fibrovascular cores. Neoplastic cells in both SPC and EPC have low- to intermediate-grade nuclei and variable myoepithelial cells [5]. Invasive papillary carcinomas are exceptionally rare, and exhibit an infiltrative growth with a predominantly papillary morphology (>90\%) [6].

Myoepithelial markers have limited utility in assigning invasive or in situ terminologies to SPC and EPC, as myoepithelial cells are absent in $80 \%$ of cases [4]. According to the World Health Organization (WHO) working group [6], in the absence of conventional invasion in SPC and EPC or infiltrative growth of SPC, both tumor types should be staged as carcinoma in situ due to their indolent clinical behavior. Studies suggest that SPC and EPC have a low incidence of lymph node metastasis and rare local or distant recurrences $[3,7,8]$. Nevertheless, the lack of myoepithelial cells is intriguing and has led to the proposal, by some authors, that these lesions are invasive carcinomas with an expansile growth pattern [9-11]. Consequently, SPC and EPC remain poorly understood entities with overlapping morphological features, which may pose challenges in classification and clinical management. In this study, we set out to describe clinical-pathologic features of SPC and EPC, including basement membrane (BM) studies.

\section{Materials and Methods}

\section{Patient Cohort}

This study was approved by the Institutional Research Ethics Board. We retrospectively identified patients diagnosed with SPC and EPC in excision or mastectomy specimens between January
2000 and September 2019, including both in-house and consultation cases. Tumors with available slides and paraffin blocks were selected. All archival hematoxylin-eosin (H \& E)-stained and immunohistochemical slides were reviewed by a breast pathologist to confirm the diagnosis. EPC was defined as solitary or multiple nodules composed of well-defined fibrovascular cores lined by monomorphic neoplastic epithelial cells with low- to intermediate-grade nuclei and surrounded by a thick fibrous capsule. SPC was defined as a lesion composed of closely apposed expansile solid nodules with delicate or inconspicuous fibrovascular cores. SPCs were categorized as invasive, if the nodules were devoid of myoepithelial cells, and had ragged contours creating a jigsaw pattern within a desmoplastic stroma [6]. Mucinous and neuroendocrine features were noted when present. Tumors were reclassified when the WHO morphological criteria (as described above) were not fulfilled. Some cases diagnosed initially as SPC or EPC were excluded from the study if the appearances fell short of either of these entities.

The following clinical-pathological features were recorded for each case: patient age at diagnosis, gender, clinical presentation, laterality (right, left, and bilateral), imaging findings, biopsy diagnosis, surgical excision procedure, the tumor size, other treatment modalities (radiotherapy and endocrine therapy), the lymph node status, follow-up duration, and status at last follow-up.

Immunohistochemistry and Fluorescent in situ Hybridization

Immunohistochemical stains were performed on $4 \mu \mathrm{m}$ thick formalin-fixed paraffin-embedded tissue sections as per the manufacturer's instructions: (a) BM markers - collagen IV (mouse monoclonal, clone CIV22, Dako) and laminin (mouse monoclonal, clone 4C7, Novus Biologicals); (b) myoepithelial cells - p63 (mouse monoclonal, clone 4A4, Roche/Ventana) and smooth muscle myosin heavy chain (SMMHC; mouse monoclonal, clone SMMS.1, Dako); and (c) biomarkers - estrogen receptor (rabbit monoclonal, clone SP1, Roche/Ventana), progesterone receptor (mouse monoclonal, clone 1294, Dako), and HER2 (rabbit monoclonal, clone 4B5, Roche/Ventana). When available, immunostains for chromogranin A (mouse monoclonal, clone LK2H10, Roche/Ventana) and synaptophysin (mouse monoclonal, clone 27G12, Novocastra) were also reviewed.

In 5 tumors with equivocal (2+) immunohistochemical staining, HER2 fluorescent in situ hybridization was performed using a dual fluorescence kit, PathVysion (Abbott Vysis Probes, Abbott Laboratories, Chicago, IL, USA) to assess the HER2 gene amplification status. Collagen IV and laminin were interpreted as present when staining of any intensity, continuous, or discontinuous, was seen at the BM surrounding the fibrovascular cores or the tumor periphery with appropriately staining internal controls and/or external controls. Positive immunoreactivity for collagen IV and laminin around blood vessels and mammary ducts served as a positive internal control.

\section{Statistical Analysis}

Statistical analysis was performed using SPSS v.26 (IBM, Inc.). Continuous variables were compared using the independent samples $t$ test or one-way ANOVA with post hoc comparison using the Sidak method. Categorical variables were compared using the $\chi^{2}$ test or Fisher's exact test, if the expected cell count was $<5$. Statistical significance was defined as $p<0.05$. 


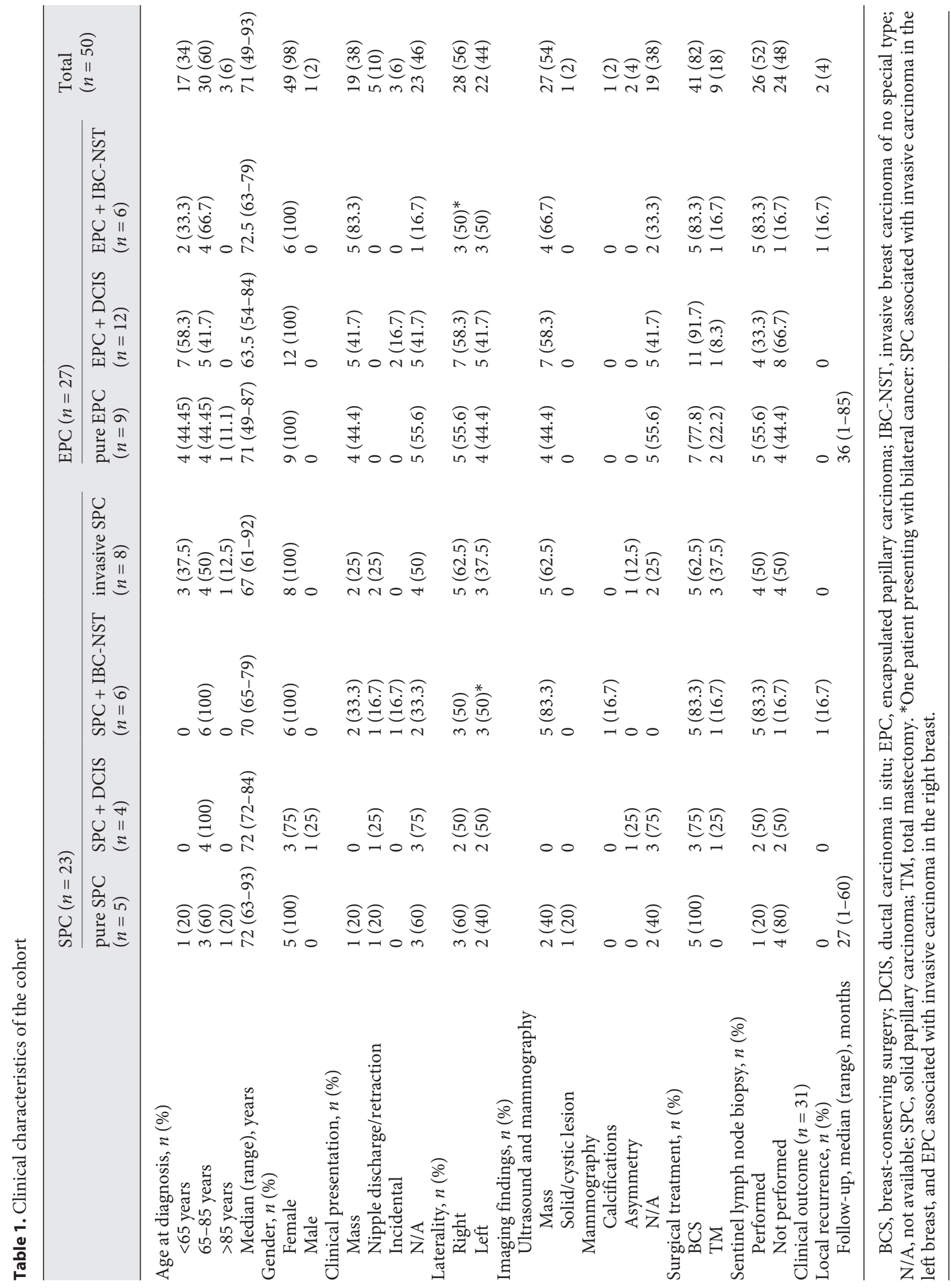


Table 2. Summary of clinicopathologic features based on histologic subtypes of SPC and EPC

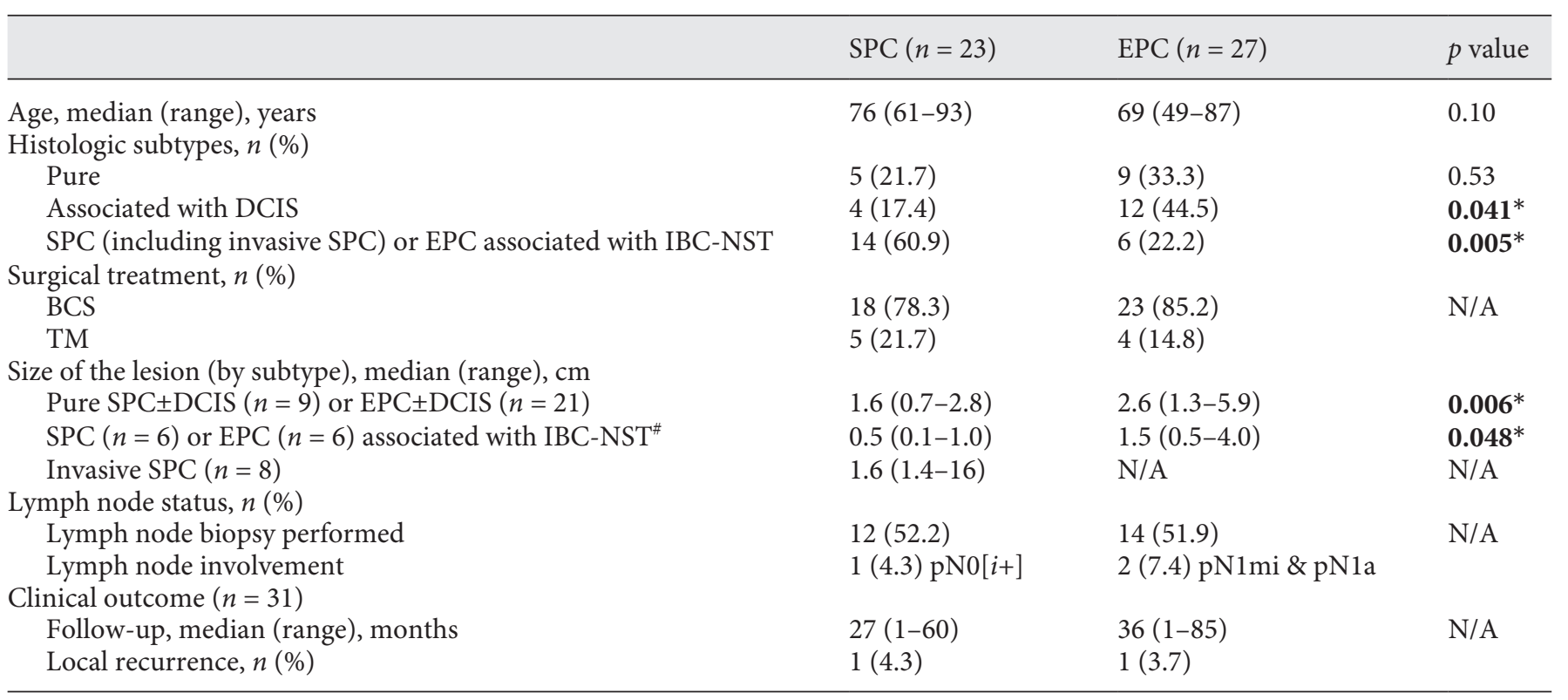

DCIS, ductal carcinoma in situ; EPC, encapsulated papillary carcinoma; IBC-NST, invasive breast carcinoma of no special type; N/A, not applicable; SPC, solid papillary carcinoma; TM, total mastectomy; BCS, breast-conserving surgery. *Statistically significant. ${ }^{\#}$ Size refers to invasive component.

\section{Results}

\section{Clinical Characteristics}

The study cohort consists of 50 tumors from 49 patients, including 23 SPCs and 27 EPCs (Tables 1,2). All tumors were unilateral, except for 1 patient who had a bilateral total mastectomy (TM) for EPC associated with invasive breast carcinoma of no special type (IBC-NST) in the right breast, and SPC associated with DCIS and mucinous carcinoma in the left breast. The median patient age was 71 years (range 49-93), with no significant difference between SPC and EPC (76 vs. 69). All patients were female except for 1 male patient with SPC. Clinical presentation was variable, the most common being a selfidentified mass in 19 patients (38\%). The most frequent imaging abnormality was also a mass lesion identified in 27 patients (54\%), including 12 SPCs and 15 EPCs.

Surgical procedures included TM in 9 patients $(18 \%)$ and breast-conserving surgery (BCS) in 41 patients (82\%), including 5 TMs and 18 BCS for patients with SPC, and 4 TMs and 23 BCS for patients with EPC. BCS was followed by radiation therapy in all cases, except for 2 patients with SPC who declined further therapy and 1 patient with EPC per tumor board consensus. Sentinel lymph node biopsy was performed in 26 cases (52\%).
Clinical outcome was available for 31 patients. At the last follow-up visit (median follow-up 35 months, range $1-85)$, most women $(29 / 31,93.5 \%)$ were free of disease. Only 2 patients, 1 with SPC (3.2\%) and another with EPC (3.2\%), both associated with IBC-NST, developed local recurrence. No distant recurrence or cancer-related deaths were observed.

\section{Pathologic Characteristics}

The tumors were classified based on the presence or absence of associated DCIS and invasive carcinoma and included 5/23 (21.7\%) pure SPCs (Fig. 1), 4/23 (17.4\%) SPCs with DCIS, 6/23 SPCs (26.1\%) associated with invasive carcinoma (Fig. 2), 8/23 (34.8\%) invasive SPCs (Fig. 3), 9/27 (33.3\%) pure EPCs, 12/27 (44.5\%) EPCs with DCIS (Fig. 4), and 6/27 (22.2\%) EPCs associated with IBC-NST (Fig. 5). Of 6 SPCs with invasive carcinoma, 1 had associated mucinous carcinoma (pure mucinous carcinoma representing $>90 \%$ of the invasive component) (Fig. 6), 3 IBC-NSTs, and 2 microinvasive carcinomas. In addition, 7 SPCs showed mucinous features (3 invasive SPCs, 2 pure SPCs, 1 SPC associated with DCIS, and 1 SPC associated with IBC-NST) and 2 invasive SPCs showed obvious neuroendocrine features. IBC-NSTs were of Nottingham histologic grade of $1-2 / 3$ and nega- 


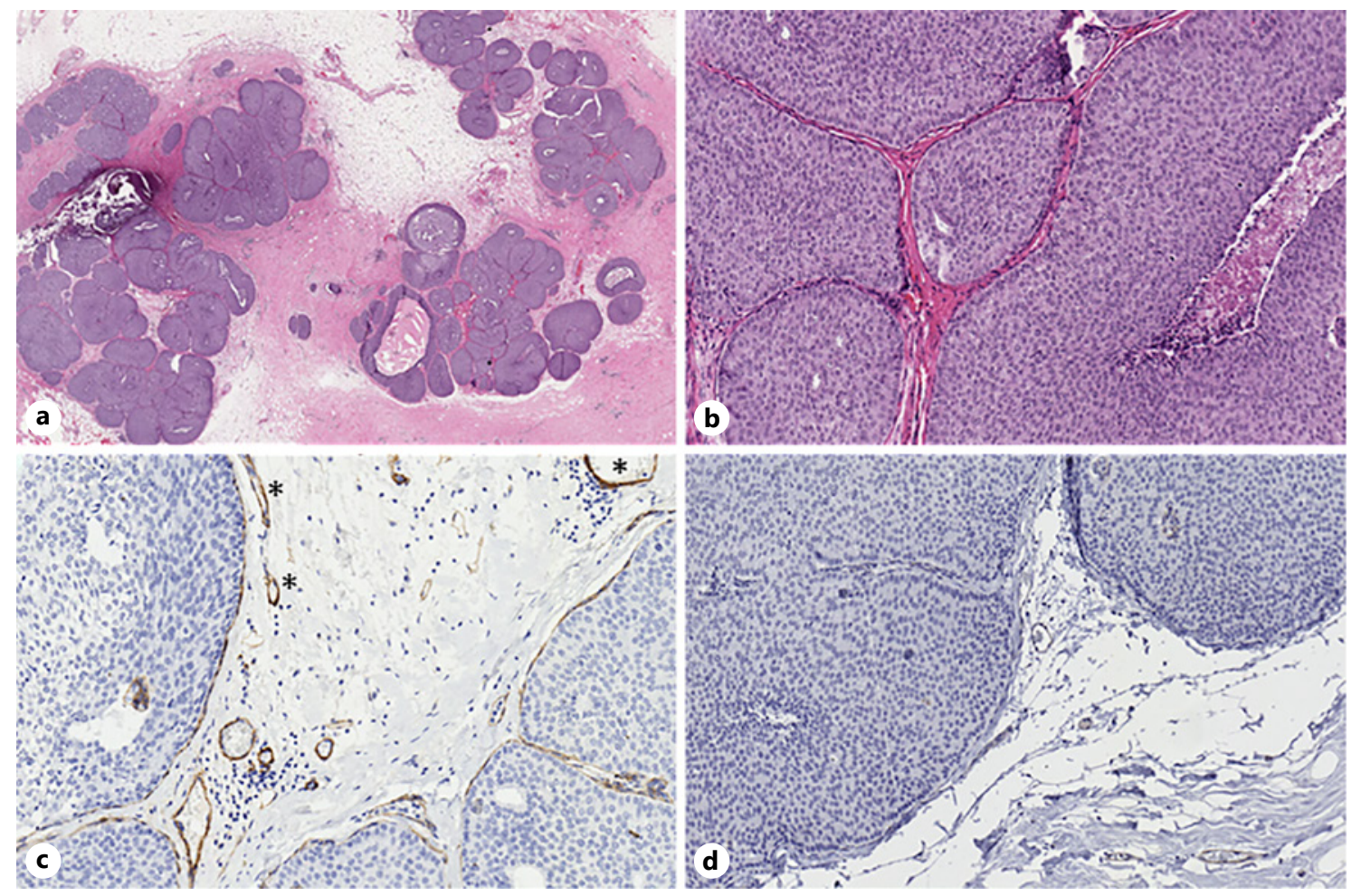

Fig. 1. Pure SPC, composed of multiple closely apposed nodules without stromal invasion (a, b), positive for collagen IV (c), and negative for laminin (d). * Blood vessels. SPC, solid papillary carcinoma.

tive for lymphovascular invasion, while DCIS showed low- to intermediate-nuclear grade.

Overall, most tumors $(30 / 50,60 \%)$ were staged as pTis; the remaining 20 tumors (including invasive SPCs as well as EPCs and SPCs associated with IBC-NST) were staged based on the size of the invasive component ranging from pT1mi to pT3 (Table 3). The invasive component in SPCs with IBC-NST was smaller than that of EPC with IBCNST ( 0.5 vs. $1.5 \mathrm{~cm} ; p=0.048$ ) (Tables 2,3 ). Similarly, the size of SPC (with or without DCIS) was smaller than that of EPC (with or without DCIS) (1.6 vs. $2.6 \mathrm{~cm} ; p=0.006$ ) (Table 2). Invasive SPCs showed the largest size variation (Table 3). SPCs were more commonly associated with invasive carcinoma (both invasive SPC and associated IBCNST) compared to EPCs (60.9 vs. $22.2 \%$; $p=0.005)$. However, EPCs were more commonly associated with DCIS ( 44.5 vs. $17.4 \% ; p=0.041$ ) (Table 2 ).

Of 26 patients who underwent sentinel lymph node biopsy, nodal involvement was identified in 3 cases $(11.5 \%)$, including 1 macrometastasis in 1 node in 1 patient with EPC (associated with IBC-NST), 1 micrometastasis in 1 node in 1 patient with EPC (associated with DCIS), and isolated tumor cells in 1 node in 1 patient with invasive SPC (Fig. 3; Tables 2, 3). Presurgical biopsy findings were available for review in 41 cases (82\%) and included "invasive carcinoma with papillary features," "atypical papillary lesion, cannot rule our carcinoma" and “ADH/DCIS” (Table 3).

\section{Immunohistochemical Features}

All tumors were positive for hormone receptors and negative for HER2 protein overexpression or gene amplification. Synaptophysin was positive in 13/23 (56.5\%) SPCs, including $75 \%$ (6/8) of invasive SPCs and 3/6 (50\%) of SPCs with invasive carcinoma. Chromogranin A was positive in $7 / 23$ (30.4\%) SPCs, including 25\% (2/8) of invasive SPCs and $2 / 6(33.3 \%)$ of SPCs with invasive carcinoma (Table 3; Fig. 6). Whenever the SPC/EPC component was positive for neuroendocrine markers, the associated invasive component also showed neuroendocrine differentiation. Most tumors were diffusely positive for both markers, except for 3 tumors with focal synaptophysin expression and 2 tumors with focal chromogranin A expression.

Of 21 patients with EPC (with or without DCIS), p63 and SMMHC were present in $8(38.1 \%)$ and $10(47.6 \%)$ 

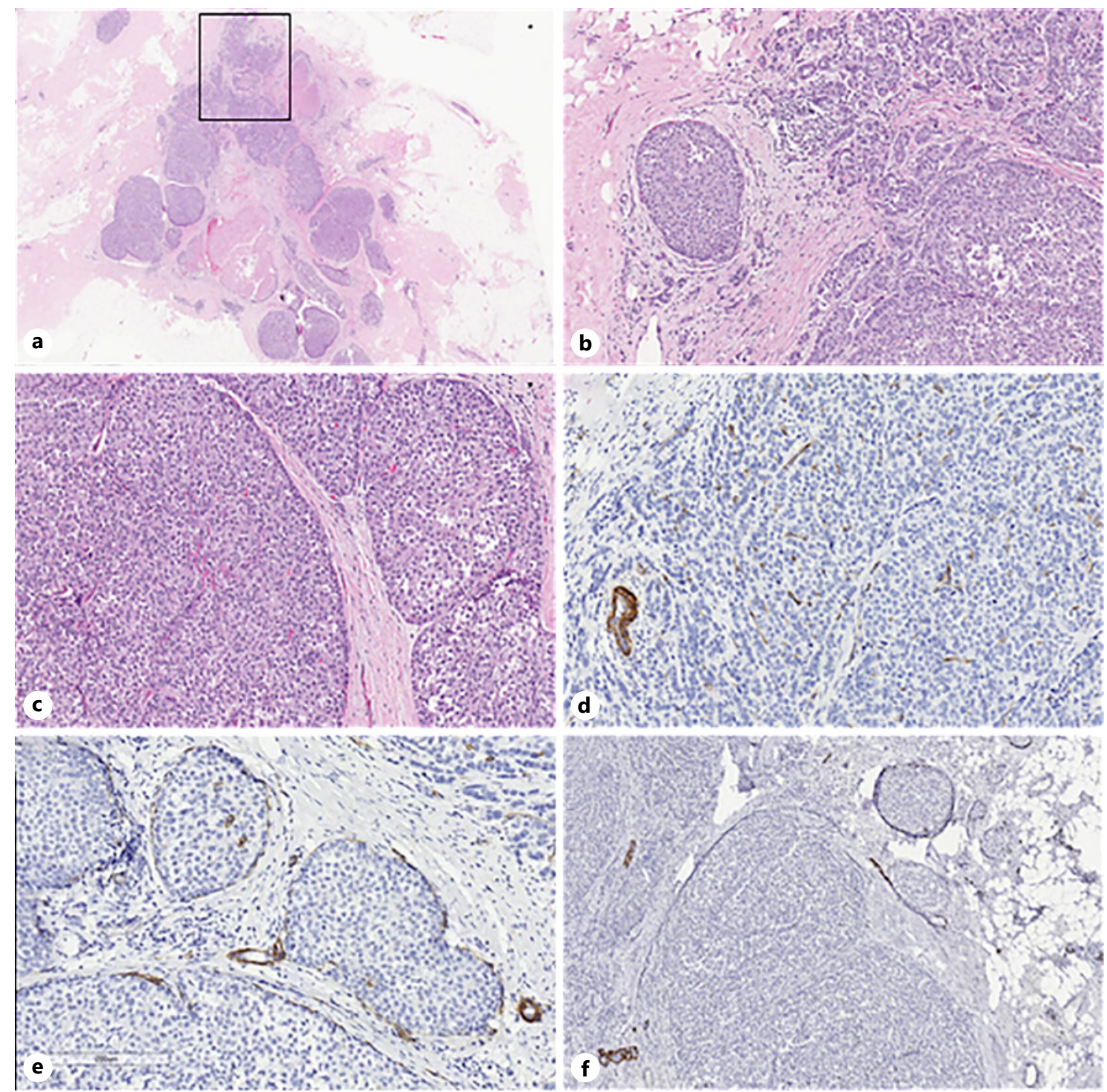

Fig. 2. SPC with IBC-NST (a, b), composed of multiple nodules with delicate fibrovascular cores (c), and associated with a focus of infiltrating nests of cells consistent with IBC-NST (inset in $\mathbf{a} ; \mathbf{b}$ ). Collagen IV is negative in IBC-NST (d) and positive in SPC (e), while p63 is present focally in SPC (f). SPC, solid papillary carcinoma; IBCNST, invasive breast carcinoma of no special type.

of tumors, respectively (Table 4). Of 23 SPCs, SMMHC was present in $100 \%$ of pure SPCs compared to $25 \%$ of SPC with DCIS and $35.7 \%$ of SPCs with IBC-NST and invasive SPCs ( $p=0.025)$ (Table 5). Differential staining of p63 in pure SPCs, SPCs with DCIS, and SPCs (with IBC-NST and invasive SPCs) did not reach statistical significance $(p=0.18)$ (Table 5). When comparing SPCs and EPCs, SMMHC was present in 5/5 (100\%) pure SPCs and only $5 / 9(55.6 \%)$ pure EPCs. In addition, p63 was present in 3/5 (60\%) pure SPCs, 6/9 (66.7\%) pure EPCs, 3/14 (21.4\%) SPCs associated with IBC-NST and invasive
SPCs, and 2/6 (33.3\%) of EPC associated with IBC-NST (Tables 3, 5). Myoepithelial marker expression was predominantly interrupted/partial, with only rare cases (SMMHC in 3 EPCs and 1 SPC) showing continuous/ circumferential staining.

Collagen IV was absent in most $(58.3 \%, 7 / 12)$ SPCs and EPCs associated with invasive carcinoma (Fig. 2, 5, 6 ) and was present in $21 / 21$ (100\%) EPCs with or without DCIS (Fig. 4) and 16/17 (94.1\%) SPCs with or without DCIS, including $8 / 8(100 \%)$ invasive SPCs $(p<0.001)$ (Fig. 1, 3; Table 4). Laminin was absent in 8/12 (66.7\%) of 


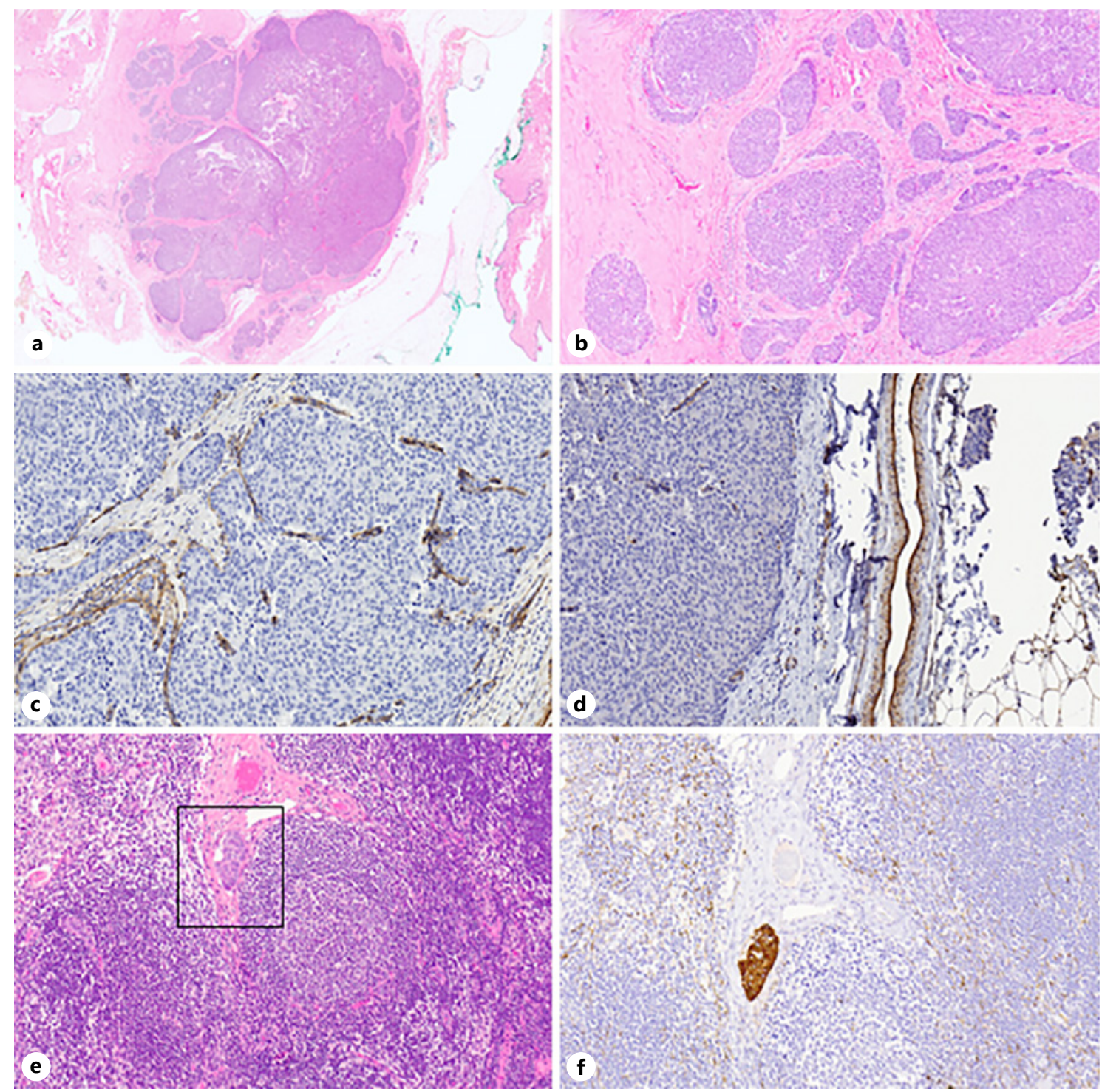

Fig. 3. Invasive SPC with irregular, jagged borders (a, b), positive for collagen IV (c), and negative for laminin (d). Isolated tumor cells are identified in one sentinel lymph node (e, inset), and are highlighted by pan-cytokeratin AE1/AE3 (f). SPC, solid papillary carcinoma.

SPCs or EPCs associated with IBC-NST (Fig. 5), and was present in 18/21 (85.7\%) EPCs with or without DCIS and $10 / 17$ (58.8\%) SPCs with or without DCIS, including 5/8 $(62.5 \%)$ invasive SPCs $(p=0.009)$ (Table 4). Overall, when comparing BM markers in SPCs and EPCs (pure, including invasive SPC, or associated with DCIS) to SPC and EPC associated with IBC-NST, collagen IV was present in $97.4 \%(37 / 38)$ versus $41.7 \%(5 / 12)$ of tumors $(p<$ $0.001)$, while laminin was present in $73.7 \%(28 / 38)$ versus $33.3 \%(4 / 12)$ of tumors $(p<0.011)$. A staining pattern of collagen IV was predominantly continuous/circumferen- tial (11 SPCs, 14 EPCs), whereas staining of laminin was mainly interrupted/partial (8 SPCs, 14 EPCs).

\section{Discussion}

SPC and EPC are rare subtypes of papillary carcinoma of the breast with distinct features and diagnostic challenges [12-15]. In this study, we report clinical-pathologic features and results of myoepithelial and BM studies in a cohort of 50 tumors from 49 patients. The median age 


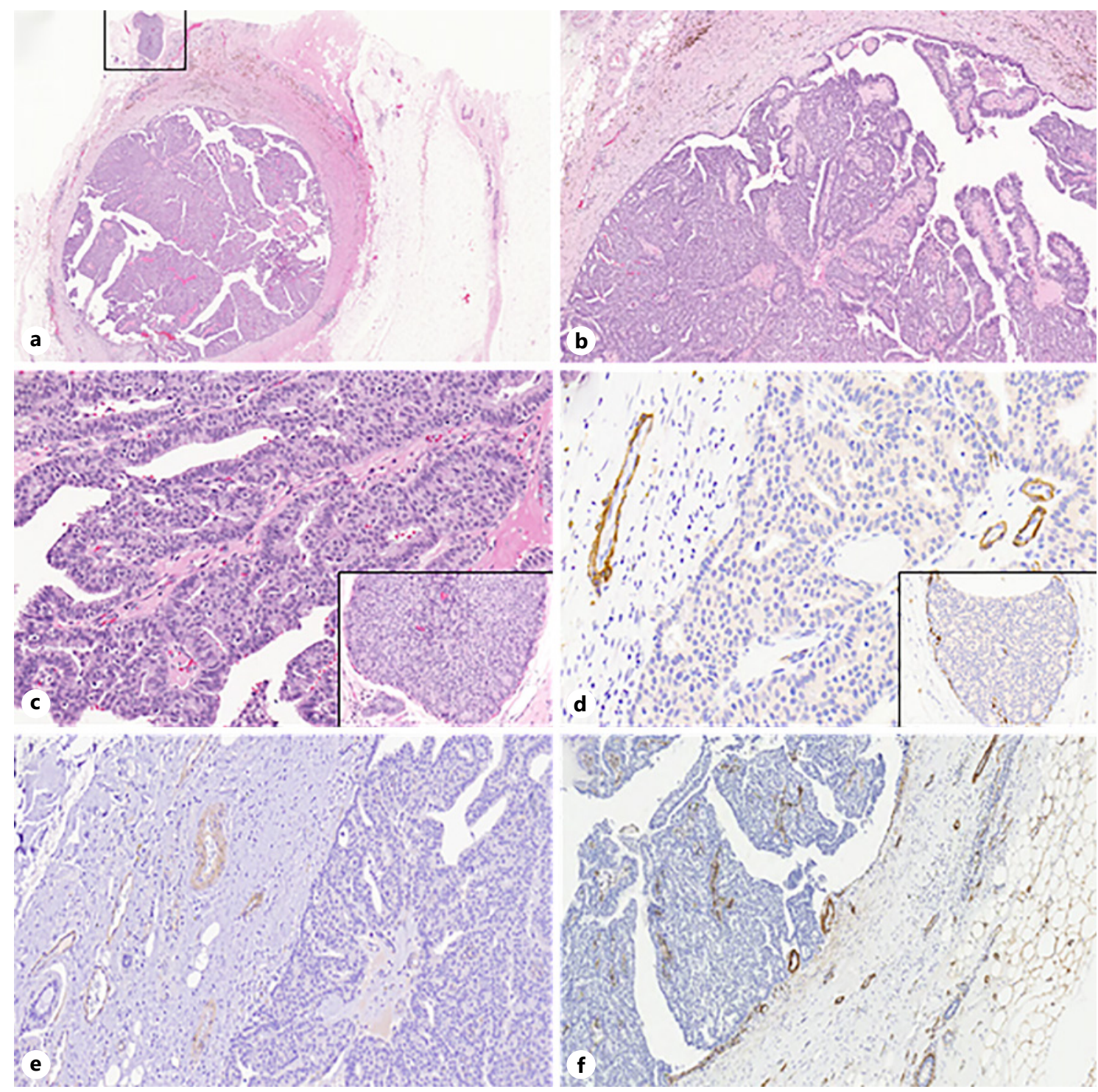

Fig. 4. EPC composed of a well-circumscribed nodule with a fibrous capsule and well-developed fibrovascular cores (a-c) and associated with conventional DCIS (insets in $\mathbf{a}, \mathbf{c})$. SMMHC is absent in EPC and present in DCIS (inset) with blood vessels serving as a positive internal control (d). Laminin is negative (e), while collagen IV is positive (f) in EPC. EPC, encapsulated papillary carcinoma; DCIS, ductal carcinoma in situ.

of patients with SPC and EPC was 76 and 69 years, respectively, in keeping with the literature [8, 14, 16-18]. Most patients were female except for 1 male patient with SPC. Other studies have also described rare cases of EPC and SPC in men $[5,19,20]$. Most patients presented with a selfidentified or radiologic mass, as reported previously [6].

Our cohort of EPCs included pure EPC (33.3\%), EPC with DCIS (44.5\%), and EPC with invasive carcinoma $(22.2 \%)$, whereas histologic subtypes of SPC were pure SPC (21.7\%), SPC with DCIS (17.4\%), SPC associated with invasive carcinoma (26.1\%), and invasive SPC (34.8\%). EPCs were more likely to be associated with DCIS compared with SPCs (44.5 vs. 17.4\%). Other studies have also shown that the majority of patients with EPC had associated conventional DCIS (outside but generally adjacent to the main lesion), IBC-NST, or both $[15,16$, 18]. Combining invasive SPCs and SPCs associated with invasive carcinoma, SPCs were more likely to be classified as invasive compared with EPC in our study (60.9 vs. 22.2\%). The highest pathological stage was pT1b for SPC 

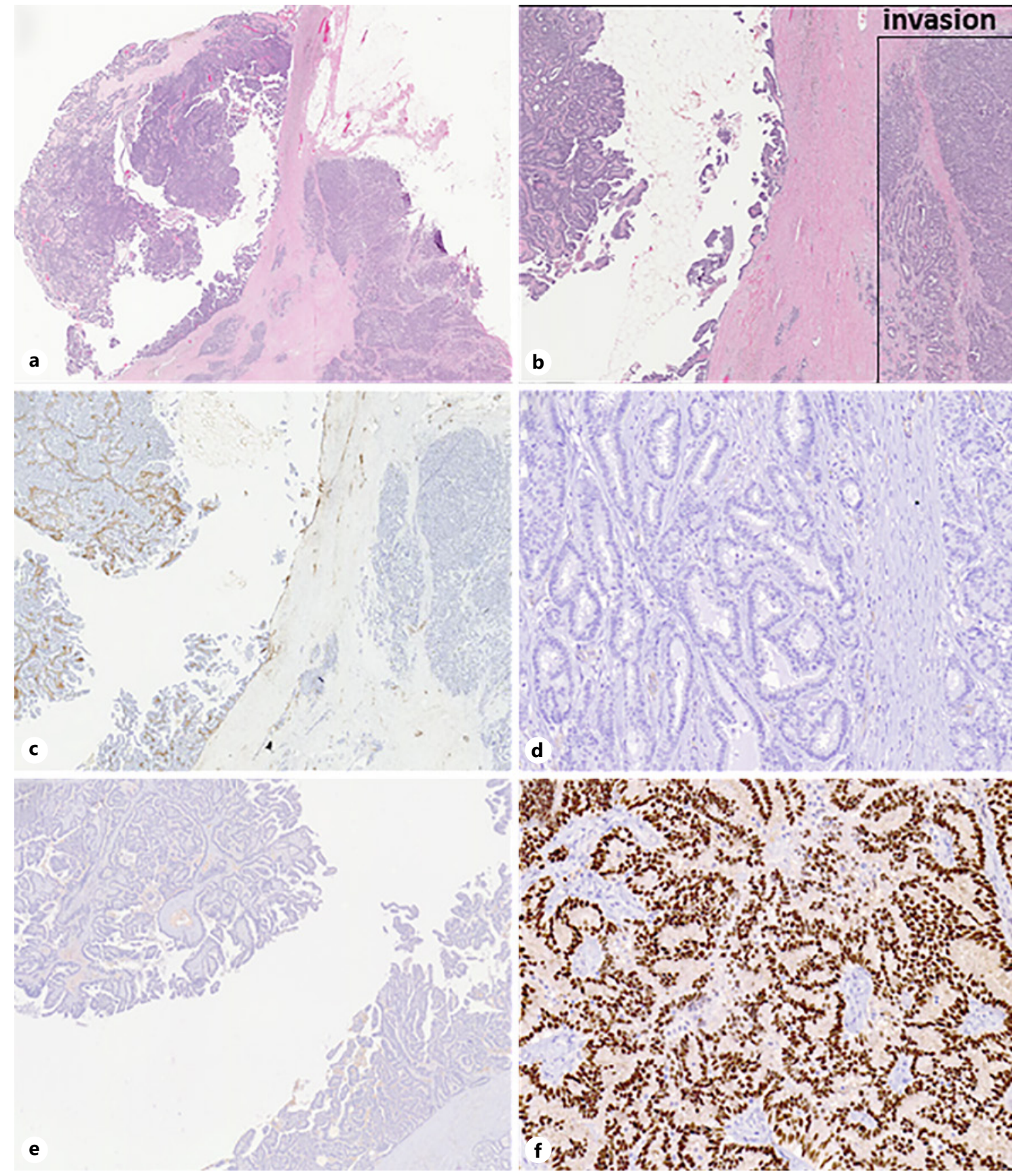

Fig. 5. EPC associated with a focus of IBC-NST (a, b). Collagen IV is positive in EPC and absent in IBC-NST (c). Laminin is absent in both EPC (not shown) and IBC-NST (d). P63 is also absent in both EPC (e) and IBC-NST (not shown). ER is strongly positive in $100 \%$ of tumor cells in EPC (f). EPC, encapsulated papillary carcinoma; IBC-NST, invasive breast carcinoma of no special type.

with invasive carcinoma, pT2 for EPC with invasive carcinoma, and pT3 for invasive SPC. This is most likely due to the fact that the presence of jagged and infiltrative borders in invasive SPC warrants classification of the entire lesion as invasive [6].

Papillary Carcinomas of the Breast
Although none of the patients developed distant recurrence or deaths, local recurrence occurred in $1 \mathrm{EPC}$ and 1 SPC $(2 / 31,6.5 \%)$, both in the context of associated invasive carcinoma. The rate of local recurrence is slightly higher in other studies [14, 16-18]. Solorzano et al. [18] 

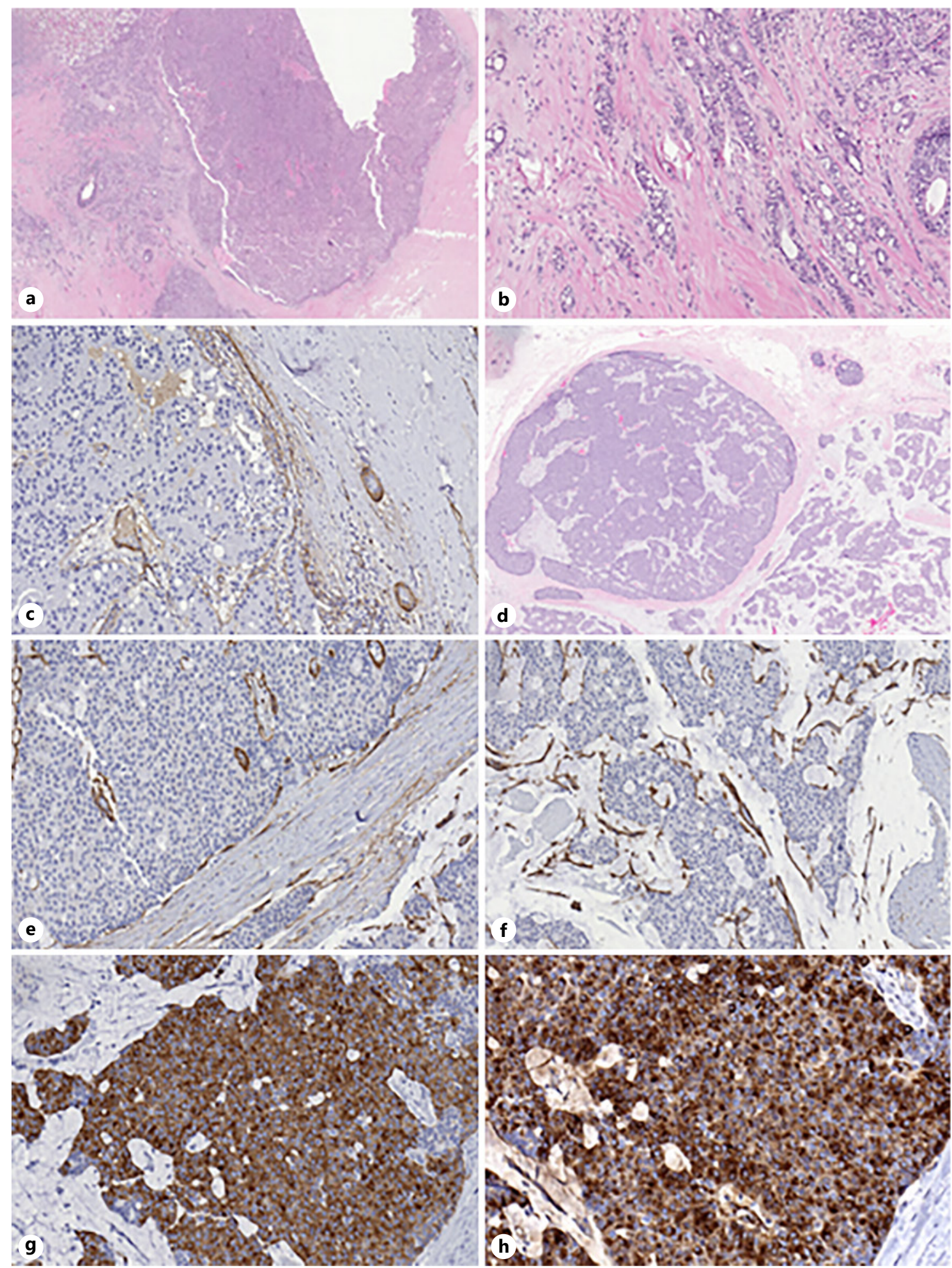

Fig. 6. The patient with bilateral mastectomy. EPC associated with a focus IBC-NST in the right breast $(\mathbf{a}, \mathbf{b})$, and concurrent SPC associated with mucinous carcinoma in the left breast (d). Collagen IV is positive in EPC (c) and SPC (e), and is negative in both invasive components (mucinous carcinoma in $\mathbf{f}$, where positivity is restricted to vascular structures). Both SPC and mucinous carcinoma are positive for synaptophysin $(\mathbf{g})$ and chromogranin A (h). SPC, solid papillary carcinoma; EPC, encapsulated papillary carcinoma; IBC-NST, invasive breast carcinoma of no special type. 


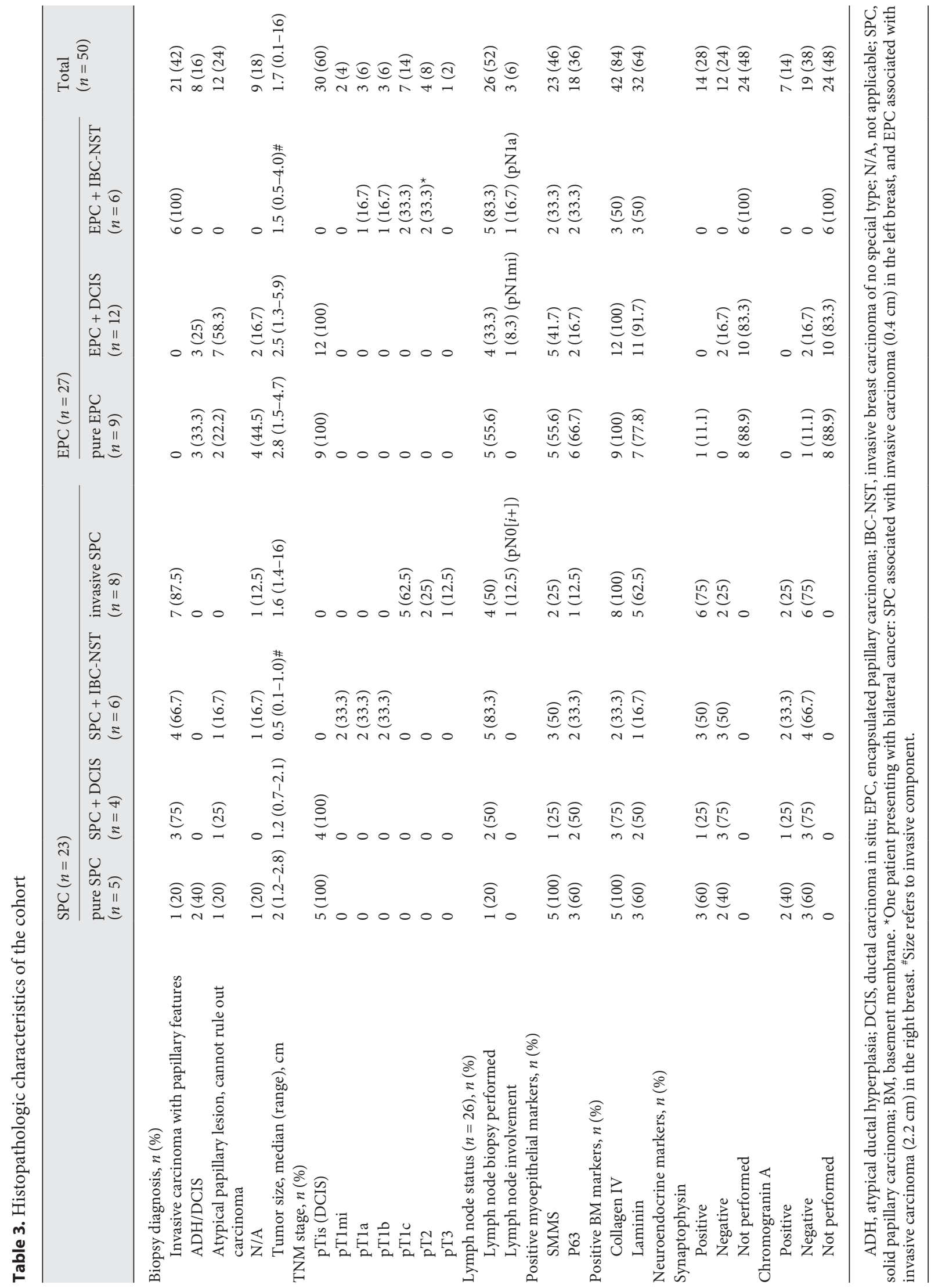


Table 4. Summary of immunohistochemistry for myoepithelial and BM markers

\begin{tabular}{llll}
\hline & $\begin{array}{l}\text { SPC and EPC with } \\
\text { IBC-NST }(n=12)\end{array}$ & $\begin{array}{l}\text { EPC with or without } \\
\text { DCIS }(n=21)\end{array}$ & $\begin{array}{l}\text { SPC (including invasive SPC) } \\
\text { with or without DCIS }(n=17)\end{array}$ \\
\hline $\begin{array}{l}\text { Positive BM markers, } n(\%) \\
\quad \text { Collagen IV }\end{array}$ & $5(41.7)$ & $21(100)$ & $16(94.1)$ \\
$\quad$ Laminin & $4(33.3)$ & $18(85.7)$ & $10(58.8)$ \\
$\begin{array}{l}\text { Positive myoepithelial markers, } n(\%) \\
\quad \text { SMMS }\end{array}$ & $5(41.7)$ & $10(47.6)$ & $8(47.1)$ \\
P63 & $4(33.3)$ & $8(38.1)$ & $6(35.3)$ \\
\hline
\end{tabular}

BM, basement membrane; DCIS, ductal carcinoma in situ; EPC, encapsulated papillary carcinoma; IBC-NST, invasive breast carcinoma of no special type; SMMS, smooth muscle myosin heavy chain; SPC, solid papillary carcinoma. ${ }^{\star S t a t i s t i c a l l y ~ s i g n i f i c a n t . ~}$

Table 5. Summary of immunohistochemistry for myoepithelial markers in patients with SPC

\begin{tabular}{llll}
\hline & $\begin{array}{l}\text { SPC, including invasive SPC or SPC associated } \\
\text { with invasive carcinoma }(n=14)\end{array}$ & $\begin{array}{l}\text { SPC with DCIS } \\
(n=4)\end{array}$ & $\begin{array}{l}\text { Pure SPC } \\
(n=5)\end{array}$ \\
\hline $\begin{array}{l}\text { Positive myoepithelial markers, } n(\%) \\
\quad \text { SMMS }\end{array}$ & $5(35.7)$ & $1(25)$ & $5(100)$ \\
P63 & $3(21.4)$ & $2(50)$ & $\mathbf{0 . 0 2 5 *}$ \\
0.18 & $3(60)$ \\
\hline
\end{tabular}

DCIS, ductal carcinoma in situ; SMMS, smooth muscle myosin heavy chain; SPC, solid papillary carcinoma. *Statistically significant.

found a $100 \%$ disease-specific survival and a recurrence rate of $12.5 \%$ in 40 EPCs. In another study of 9 EPCs and 5 SPCs, locoregional recurrence occurred only in 1 patient (7.1\%) with SPC associated with DCIS [8]. This suggests that most cases of SPC and EPC have favorable outcomes with significantly high recurrence-free survival rates $[8,21,22]$.

In our cohort, the majority of patients underwent BCS (78.3\% for SPCs, $85.2 \%$ for EPCs) followed by radiation therapy, and $52 \%$ had sentinel lymph node biopsy. In the published series of EPCs, the rates of BCS range from 29 to $93 \%[14,16-18]$. In one study, $60 \%$ of patients with SPC underwent TM compared with only $22 \%$ of patients with EPC [8]. The rate of sentinel lymph node biopsy ranges from 30 to $100 \%$ in the literature $[8,14,16-18]$, suggesting that uncertainty regarding the presence of invasion based on core needle biopsy (CNB) may be contributing to the surgeon's decision to perform a sentinel lymph node biopsy. There may also be interinstitutional variability. In addition to SPCs and EPCs associated with invasive carcinoma, we recommend routine sentinel lymph node biopsy in tumors without frank invasion on $\mathrm{CNB}$ as the presence of invasive component can never be excluded with $100 \%$ certainty based on CNB. To further support this notion, the papillary nature of the targeted lesion was appreciated in most pre-excision biopsies in our study, with $\mathrm{CNB}$ diagnoses ranging from invasive carcinoma to invasive carcinoma with papillary features or atypical papillary lesion, cannot rule out carcinoma.

Macro- or micrometastases were seen in 2 patients, 1 with EPC and IBC-NST, and the other with EPC and DCIS; 1 patient with invasive SPC also had ITCs. Although lymph node involvement is uncommon in both tumor types, it has been reported previously ranging from 0 to $11 \%[3,8,14-19,23,24]$. The finding of metastatic carcinoma in cases lacking obvious invasion can be explained by occult stromal invasion (inadequate sampling) or iatrogenic displacement of cells due to prior biopsy $[25,26]$, especially given the phenomenon of post-needling or post-manipulation seeding being more common in papillary lesions [27].

All tumors in our cohort were positive for hormone receptors and negative for HER2 as expected [6]. SPCs may exhibit neuroendocrine differentiation, although this does not appear to have a clinical significance [28]. More than half of SPCs in our study were positive for synaptophysin, and one-third was positive for chromogranin $\mathrm{A}$, including the invasive component. In practice, we rec- 
ommend reporting ER, PR, and HER2, if SPC or EPC is associated with invasive carcinoma, or SPC is classified as invasive. Similarly, if tumors are classified as pTis, only ER and PR should be reported. We do not recommend routine immunohistochemistry for neuroendocrine markers for the purpose of histotyping, especially given the fact that IBC-NST may occasionally show expression of neuroendocrine markers; however, these stains may be helpful in cases where primary and recurrent or metastatic tumors are available for comparison.

As per the WHO classification, EPCs typically lack myoepithelial cell markers within the papillae as well as at the periphery of the lesion. SPCs frequently lack myoepithelial cells at the periphery but may show focal staining [6]. Since the presence of myoepithelial cells is historically used as a main criterion for a noninvasive process, it has been postulated that EPC represents an indolent tumor with pushing borders or a minimally invasive form of low-grade carcinoma [2,9]. In our study, SMMHC was present in $100 \%$ of pure SPCs, but only in $55.6 \%$ of pure EPCs. Not surprisingly, staining was largely absent in EPCs, and SPCs with associated IBC-NST, as well as invasive SPCs. Similarly, p63 was present in $60 \%$ of pure SPCs and $66.7 \%$ of pure EPCs compared with $21.4 \%$ and $33.3 \%$ of their counterparts associated with invasion. This suggests that it is more likely to encounter negative myoepithelial markers in EPCs even in the absence of conventional invasion. Therefore, immunohistochemistry for myoepithelial markers has a limited utility in practice, especially in CNBs which often show detached fragments of papillary carcinoma, and invasion is difficult or impossible to assess (such cases may be signed out as papillary carcinoma, at least in situ). Nevertheless, these markers may be performed, if desired, but should be interpreted with caution.

Wynveen et al. [24] described complete absence of myoepithelial cells morphologically in 33/40 (82.5\%) EPCs. By immunohistochemistry (p63, calponin, SMMHC, and 34ßE12, CD10), focal positivity was seen in 7/40 (17.5\%), including pure EPCs and EPCs associated with IBC-NST. The authors concluded that there was no relationship between the presence of associated invasive carcinoma and the presence or absence of myoepithelial cells [24]. Similarly, focal peripheral myoepithelial markers, as demonstrated by p63 and SMMHC stains, were seen in 6/27 and 4/9 of pure EPCs in 2 studies $[9,29]$. Collins et al. [2] and Calderaro et al. [30] reported complete absence of myoepithelial cells in 22 EPCs (using SMMHC, calponin, p63, CD10, and cytokeratin 5/6) and 20 EPCs, including 10 cases with invasive carcinoma (using p63,

Papillary Carcinomas of the Breast
CD10, and smooth muscle actin), respectively. After the former study, these lesions were designated as encapsulated as opposed to intracystic carcinoma. Several studies have since suggested that some, if not all, EPCs represent circumscribed nodules of invasive papillary carcinoma rather than pure in situ disease, but nevertheless with an indolent clinical behavior and low metastatic potential [2, 9, 30, 31].

As for BM markers in our cohort, collagen IV was present in 18/23 (78.3\%) SPCs, including $100 \%$ of pure SPCs, $75 \%$ of SPCs with DCIS, $33.3 \%$ of SPCs with IBCNST, and $100 \%$ of invasive SPCs. Collagen IV was also positive in $24 / 27$ (88.9\%) EPCs, including $100 \%$ of pure EPCs with or without DCIS and $50 \%$ of EPCs associated with IBC-NST. The majority of tumors showed a continuous/circumferential staining pattern for collagen IV, whereas the interrupted pattern was seen in a small subset. Similarly, in a cohort of 40 EPCs, most (89\%) cases showed positivity for collagen IV, albeit discontinuous and fragmented. These included EPCs associated with IBC-NST, of which only 1 tumor was completely negative for collagen IV [24]. The authors concluded that collagen IV stain is not a reliable marker to evaluate stromal invasion in EPCs, and that a continuous layer of collagen IV does not necessarily indicate an in situ process. In another study of 81 invasive carcinomas, collagen IV staining was associated with low histologic grade, absence of nodal metastasis and improved disease-free survival [32]. In another study of 27 EPCs, collagen IV was expressed in all cases (including 6 EPCs with invasive carcinoma), and all patients were disease-free at last follow-up (mean 40 months) suggesting that EPC may be considered carcinoma in situ with excellent prognosis compared to conventional IBC-NST [29].

Based on the fact that laminin and collagen form the structural integrity of BM, we also performed immunohistochemistry for laminin, which showed staining in $85.7 \%$ of EPCs with and without DCIS and $58.8 \%$ of SPCs (including invasive SPC) with and without DCIS. In one study, DCIS showed linear well-formed laminin and collagen IV without disruptions as opposed to the striking lack of extracellular immunoreactivity for those markers in invasive ductal and lobular carcinomas [33]. Overall, the presence of collagen IV and laminin in all EPCs (pure and with DCIS) and $94.1 \%$ of SPCs (pure and with DCIS) in our study lend support to the concept that although the majority of these tumors may be considered in situ lesions, some may represent well-differentiated (lowgrade) invasive carcinomas. An interesting but confounding phenomenon in interpretation of BM immunohisto- 
chemistry is that vascular structures, not uncommonly seen at the periphery of papillary lesions as well as within fibrovascular cores, also stain for BM markers. Caution should be taken during assessment so as not to overcall intermittent positivity of vascular structures as positive epithelial staining. A helpful clue is a double BM in arteriole-type structures in contrast to long stretches of positivity, especially in between vessels of papillary lesions, pointing to true BM staining.

In conclusion, our study confirms that SPC and EPC are distinct types of papillary carcinoma with characteristic morphologic and immunophenotypic features and favorable prognosis, with 2 local recurrences occurring in the presence of invasion. SPCs are more commonly associated with invasive carcinoma or considered invasive compared to EPC (60.9 vs. $22.2 \%$ ), whereas EPCs have a significantly higher incidence of associated DCIS. Lymph node involvement can be seen in rare cases of SPC and EPC, irrespective of associated invasion. The presence of collagen IV in all EPCs and the majority of SPCs (including invasive SPC) and its absence in a substantial proportion of SPCs or EPCs associated with IBC-NST indicates that the majority of these tumors may represent in situ lesions; however, some may behave as low-grade invasive malignancy with metastatic potential even in the absence of conventional invasion.

\section{Statement of Ethics}

The research was conducted ethically in accordance with the World Medical Association Declaration of Helsinki. The study protocol was approved by the Institute's Committee on human research. The Research Ethics Board approval number is 18-0216E. As a retrospective study of archival material, the study did not require written informed consent. The article does not contain any studies with animals performed by any of the authors.

\section{Conflict of Interest Statement}

The authors have no conflicts of interest to declare.

\section{Funding Sources}

The authors did not receive any funding.

\section{Author Contributions}

S.M.: data collection, data analysis and interpretation, and drafting the article; D.D.: data analysis; J.W.: data collection; G.T.: conception of the work, data analysis and interpretation, and critical revision of the article.

\section{References}

1 Wei S. Papillary lesions of the breast: an update. Arch Pathol Lab Med. 2016 Jul;140(7):628-43.

2 Collins LC, Carlo VP, Hwang H, Barry TS, Gown AM, Schnitt SJ. Intracystic papillary carcinomas of the breast: a reevaluation using a panel of myoepithelial cell markers. Am J Surg Pathol. 2006 Aug;30(8):1002-7.

3 Rakha EA, Gandhi N, Climent F, van Deurzen $\mathrm{CH}$, Haider SA, Dunk L, et al. Encapsulated papillary carcinoma of the breast: an invasive tumor with excellent prognosis. Am J Surg Pathol. 2011 Aug;35(8):1093-103.

4 Rakha EA, Ahmed MA, Ellis IO. Papillary carcinoma of the breast: diagnostic agreement and management implications. Histopathology. 2016 Nov;69(5):862-70.

5 Saremian J, Rosa M. Solid papillary carcinoma of the breast: a pathologically and clinically distinct breast tumor. Arch Pathol Lab Med. 2012 Oct;136(10):1308-11.

6 Board WCoTE. WHO classification of tumours of the breast. Lyon, France: International Agency for Research on Cancer (IARC); 2019.

7 Leal C, Costa I, Fonseca D, Lopes P, Bento MJ, Lopes C. Intracystic (encysted) papillary carcinoma of the breast: a clinical, pathological, and immunohistochemical study. Hum Pathol. 1998 Oct;29(10):1097-104.
8 Turashvili G, Brogi E, Morrow M, Hudis C, Dickler M, Norton L, et al. The 21-gene recurrence score in special histologic subtypes of breast cancer with favorable prognosis. Breast Cancer Res Treat. 2017 Aug;165(1):65-76.

9 Hill CB, Yeh IT. Myoepithelial cell staining patterns of papillary breast lesions: from intraductal papillomas to invasive papillary carcinomas. Am J Clin Pathol. 2005 Jan;123(1): 36-44.

10 Collins LC, Schnitt SJ. Papillary lesions of the breast: selected diagnostic and management issues. Histopathology. 2008 Jan;52(1):20-9.

11 Koerner F. Papilloma and papillary carcinoma. Semin Diagn Pathol. 2010 Feb;27(1):1330.

12 Gatchell FG, Dockerty MB, Clagett OT. Intracystic carcinoma of the breast. Surg Gynecol Obstet. 1958 Mar;106(3):347-52.

13 Czernobilsky B. Intracystic carcinoma of the female breast. Surg Gynecol Obstet. 1967 Jan; 124(1):93-8.

14 Carter D, Orr SL, Merino MJ. Intracystic papillary carcinoma of the breast. After mastectomy, radiotherapy or excisional biopsy alone. Cancer. 1983 Jul 1;52(1):14-9.

15 Mulligan AM, O’Malley FP. Metastatic potential of encapsulated (intracystic) papillary carcinoma of the breast: a report of 2 cases with axillary lymph node micrometastases. Int J Surg Pathol. 2007 Apr;15(2):143-7.

16 Lefkowitz M, Lefkowitz W, Wargotz ES. Intraductal (intracystic) papillary carcinoma of the breast and its variants: a clinicopathological study of 77 cases. Hum Pathol. 1994 Aug; 25(8):802-9.

17 Harris KP, Faliakou EC, Exon DJ, Nasiri N, Sacks NP, Gui GP. Treatment and outcome of intracystic papillary carcinoma of the breast. Br J Surg. 1999 Oct;86(10):1274.

18 Solorzano CC, Middleton LP, Hunt KK, Mirza N, Meric F, Kuerer HM, et al. Treatment and outcome of patients with intracystic papillary carcinoma of the breast. Am J Surg. 2002 Oct; 184(4):364-8.

19 Nassar H, Qureshi H, Adsay NV, Volkanadsay N, Visscher D. Clinicopathologic analysis of solid papillary carcinoma of the breast and associated invasive carcinomas. Am J Surg Pathol. 2006 Apr;30(4):501-7.

20 Yoshida M, Mouri Y, Yamamoto S, Yorozuya K, Fujii K, Nakano S, et al. Intracystic invasive papillary carcinoma of the male breast with analyses of loss of heterozygosity on chromosome 16q. Breast Cancer. 2010 Apr;17(2): 146-50. 
21 Rosen PP, Groshen S, Kinne DW, Norton L. Factors influencing prognosis in node-negative breast carcinoma: analysis of 767 T1N0M0/T2N0M0 patients with long-term follow-up. J Clin Oncol. 1993 Nov;11(11): 2090-100.

22 Otsuki Y, Yamada M, Shimizu S, Suwa K, Yoshida M, Tanioka F, et al. Solid-papillary carcinoma of the breast: clinicopathological study of 20 cases. Pathol Int. 2007 Jul;57(7): 421-9.

23 Nicolas MM, Wu Y, Middleton LP, Gilcrease MZ. Loss of myoepithelium is variable in solid papillary carcinoma of the breast. Histopathology. 2007 Nov;51(5):657-65.

24 Wynveen CA, Nehhozina T, Akram M, Hassan M, Norton L, Van Zee KJ, et al. Intracystic papillary carcinoma of the breast: an in situ or invasive tumor? Results of immunohistochemical analysis and clinical follow-up. Am J Surg Pathol. 2011 Jan;35(1):1-14.
25 Carter BA, Jensen RA, Simpson JF, Page DL. Benign transport of breast epithelium into axillary lymph nodes after biopsy. Am J Clin Pathol. 2000 Feb;113(2):259-65.

26 Nagi C, Bleiweiss I, Jaffer S. Epithelial displacement in breast lesions: a papillary phenomenon. Arch Pathol Lab Med. 2005 Nov; 129(11):1465-9.

27 Bleiweiss IJ, Nagi CS, Jaffer S. Axillary sentinel lymph nodes can be falsely positive due to iatrogenic displacement and transport of benign epithelial cells in patients with breast carcinoma. J Clin Oncol. 2006 May 1;24(13): 2013-8.

28 Tan PH, Schnitt SJ, van de Vijver MJ, Ellis IO, Lakhani SR. Papillary and neuroendocrine breast lesions: the WHO stance. Histopathology. 2015 May;66(6):761-70.

29 Esposito NN, Dabbs DJ, Bhargava R. Are encapsulated papillary carcinomas of the breast in situ or invasive? A basement membrane study of 27 cases. Am J Clin Pathol. 2009 Feb; 131(2):228-42.
30 Calderaro J, Espie M, Duclos J, Giachetti S, Wehrer D, Sandid W, et al. Breast intracystic papillary carcinoma: an update. Breast J. 2009 Nov-Dec;15(6):639-44.

31 Mulligan AM. Encapsulated papillary carcinoma of the breast. Surg Pathol Clin. 2009 Jun;2(2):319-50.

32 Visscher DW, Sarkar FH, Sakr W, Crissman J. Immunohistologic analysis of invasive phenotype in breast carcinoma. A clinicopathologic study. Pathol Res Pract. 1993 Sep;189(8): $867-72$.

33 Maatta M, Virtanen I, Burgeson R, AutioHarmainen $\mathrm{H}$. Comparative analysis of the distribution of laminin chains in the basement membranes in some malignant epithelial tumors: the alpha1 chain of laminin shows a selected expression pattern in human carcinomas. J Histochem Cytochem. 2001 Jun; 49(6):711-26. 